\title{
Human noncoding RNA 886 (nc886) adopts two structurally distinct conformers that are functionally opposing regulators of PKR
}

\author{
BRENDA M. CALDERON ${ }^{1,2}$ and GRAEME L. CONN ${ }^{1}$ \\ ${ }^{1}$ Department of Biochemistry, Emory University School of Medicine, Atlanta, Georgia 30322, USA \\ ${ }^{2}$ Graduate Program in Biochemistry, Cell and Developmental Biology (BCDB), Emory University, Atlanta, Georgia 30322 USA
}

\begin{abstract}
The double-stranded RNA (dsRNA)-activated protein kinase (PKR) senses dsRNA produced during viral infection and halts cellular protein synthesis to block viral replication. How basal PKR activity is controlled in the absence of infection was unclear until the recent identification of a potential endogenous regulator, the cellular noncoding RNA 886 (nc886). However, nc886 adopts two distinct conformations for which the structural details and potential functional differences remain unclear. Here, we isolated and separately dissected the function of each form of nc886 to more clearly define the molecular mechanism of nc886-mediated PKR inhibition. We show that nc886 adopts two stable, noninterconverting RNA conformers that are functionally nonequivalent using complementary RNA structure probing and mutational analyses combined with PKR binding and activity assays. One conformer acts as a potent inhibitor, while the other is a pseudoinhibitor capable of weakly activating the kinase. We mapped the nc886 region necessary for high affinity binding and potent inhibition of PKR to an apical stem-loop structure present in only one conformer of the RNA. This structural feature is not only critical for inhibiting PKR autophosphorylation, but also the phosphorylation of its cellular substrate, the eukaryotic translation initiation factor $2 \alpha$ subunit. The identification of different activities of the nc886 conformers suggests a potential mechanism for producing a gradient of PKR regulation within the cell and reveals a way by which a cellular noncoding RNA can mask or present a structural feature to PKR for inhibition.
\end{abstract}

Keywords: noncoding RNA; RNA structure; translational control; elF2 $\alpha$; kinase

\section{INTRODUCTION}

The innate immune response is an intrinsic cellular system that serves as the first defense against pathogens (Schoggins and Rice 2011; Iwasaki 2012). Pattern recognition receptors (PRRs) on the cell surface and in the cytosol detect pathogen-associated molecular patterns, such as viral proteins and nucleic acids, and initiate signaling cascades to prevent viral replication and to establish an antiviral state (Yoneyama and Fujita 2010; Dalet et al. 2015; Schlee and Hartmann 2016). One critical PRR is the double-stranded RNA (dsRNA)activated protein kinase (PKR), which senses dsRNA in the cytoplasm and halts protein synthesis (García et al. 2006, 2007; Balachandran and Barber 2007; Dalet et al. 2015). Binding of viral dsRNA promotes PKR dimerization and autophosphorylation, activating the kinase to phosphorylate its substrate, the eukaryotic translation initiation factor $2 \alpha$ subunit $(\mathrm{eIF} 2 \alpha)$ on serine 51. Phosphorylation increases eIF2 affinity for its guanine nucleotide exchange factor eIF2B and thus sequesters the protein complex, effectively

Corresponding author: gconn@emory.edu

Article is online at http://www.rnajournal.org/cgi/doi/10.1261/rna.060269. 116. halting recycling of eIF2 to a GTP-bound form (Sudhakar et al. 2000). As a result, general translation is attenuated, blocking viral replication while permitting enhanced translation of specific transcripts including stress response genes (Yamasaki and Anderson 2008).

The central importance of PKR to innate immunity is highlighted by the diverse array of mechanisms viruses have evolved to subvert this pathway (Langland et al. 2006; McKenna et al. 2007; Domingo-Gil et al. 2011). Due to its ubiquitous expression and functional roles outside of innate immunity, PKR activity must be tightly regulated so that it can recognize viral RNA, but not be spuriously activated by cellular RNA. Studies on the proliferative role of PKR in cancer cells recently led to the discovery of a potential endogenous RNA regulator, the noncoding RNA 886 (nc886) (Fig. 1A; Lee et al. 2011). nc886 is a ubiquitously expressed, cytosolic noncoding RNA that was found to interact with PKR

C 2017 Calderon and Conn This article is distributed exclusively by the RNA Society for the first 12 months after the full-issue publication date (see http://rnajournal.cshlp.org/site/misc/terms.xhtml). After 12 months, it is available under a Creative Commons License (Attribution-NonCommercial 4.0 International), as described at http://creativecommons.org/licenses/ by-nc/4.0/. 


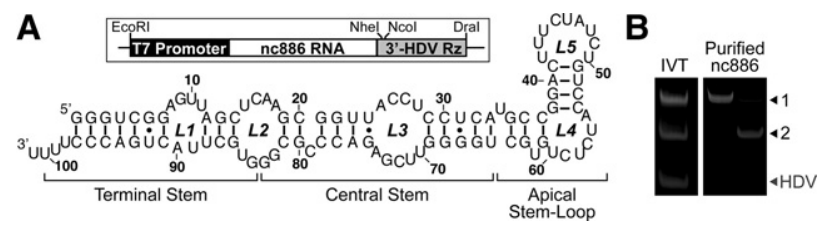

FIGURE 1. nc886 RNA forms two distinct, noninterconverting conformers that can be isolated and characterized separately. (A) The predicted secondary structure of nc886 derived using Mfold software (Zuker 2003), with the three regions of the RNA referred to in the main text indicated below. Inset, schematic of the construct used to produce the nc886-HDV ribozyme in vitro transcript. (B) Native PAGE analysis of the in vitro transcription reaction (IVT) and purified nc886 conformers stained with ethidium bromide.

(Lee et al. 2011). Additionally, expression of nc886 is downregulated or completely absent in many cancer cells along with associated increased levels of PKR phosphorylation (Treppendahl et al. 2011; Xiong et al. 2011; Kunkeaw et al. 2012; Cao et al. 2013; Lee et al. 2014a,b; Romanelli et al. 2014; Lee 2015). Silencing of nc886 leads to increased basal kinase activity, as demonstrated by PKR autophosphorylation of threonine 446 and phosphorylation of eIF $2 \alpha$ in the absence of exogenous activating dsRNA (Lee et al. 2011; Kunkeaw et al. 2012). These observations clearly point to the existence of cellular RNAs with the ability to activate PKR in the absence of nc886. Furthermore, reintroduction of nc886 to cells lacking the transcript resulted in reduced levels of phosphorylated PKR and eIF2 $\alpha$, demonstrating that nc886 is necessary and sufficient to negatively regulate PKR activity (Kunkeaw et al. 2012).

Despite this significant advance in our understanding of endogenous regulation of PKR activity, many questions remain about the nature of nc886-mediated PKR inhibition. In particular, it remains unclear why nc 886 behaves as an inhibitor rather than an activator of PKR activity and what specific RNA features confer its potency of inhibition. Notably, although nc886 was found to exist in two conformations, all analyses performed to date used a mixture of these two different forms. We therefore set out to isolate and separately dissect the functions of these two conformers to clearly define the molecular mechanisms of nc886-mediated PKR inhibition.

Our results demonstrate that nc886 RNA does indeed exist in two structurally and functionally nonequivalent forms. One conformer, which exhibits slower migration on native polyacrylamide gels, has higher binding affinity for PKR and potently inhibits both PKR and downstream eIF2 $\alpha$ phosphorylation. In contrast, the second conformer is a weak activator that behaves as a pseudoinhibitor of PKR in competition with other dsRNAs, and may serve as a more transient regulator of PKR activity. This work emphasizes the importance of RNA folding in regard to noncoding RNA function and highlights a potential mechanism for regulating the activity of nc886 RNA independent of its transcript level through the presentation or masking of a critical structural element.

\section{RESULTS}

nc886 RNA adopts two noninterconverting conformers with distinct stabilities

nc886 RNA (101 nucleotides) was in vitro transcribed using T7 RNA polymerase from a linearized plasmid DNA template producing nc886 fused at its $3^{\prime}$-end to a self-cleaving hepatitis delta virus (HDV) ribozyme (Fig. 1A). The in vitro transcription reaction was analyzed by native polyacrylamide gel electrophoresis (PAGE) revealing two nc886 RNA bands of differing mobility, as previously reported (Jeon et al. 2012), in addition to the cleaved HDV ribozyme (Fig. 1B). These distinct nc886 structural conformers are referred to as "Conformer 1" (slower migrating form) and "Conformer 2" (faster migrating form).

Purification of nc 886 by denaturing PAGE results in a mixture of both conformers (Jeon et al. 2012). We therefore used preparative native PAGE to isolate the individual nc886 RNA conformers and found through reanalysis of the purified conformers that both structures are stable and do not interconvert under nondenaturing conditions (Fig. 1B). We next analyzed the thermal stability of each isolated nc886 RNA conformer by UV melting analysis in the same $0.5 \times \mathrm{TBE}$ buffer as used in native PAGE (Fig. 2A). The melting profile (first derivative of the raw UV melting curve) for nc886 Conformer 2 revealed that this RNA structure unfolds in a single apparent transition with a $T_{\mathrm{m}}$ of $\sim 40^{\circ} \mathrm{C}$. In contrast, Conformer 1 exhibits much greater stability with a final major apparent unfolding transition with a $T_{\mathrm{m}}>70^{\circ} \mathrm{C}$. Unfolding of each nc886 conformer was also assessed in 20 mM HEPES buffer ( $\mathrm{pH} 7.5$ ) containing $100 \mathrm{mM} \mathrm{NaCl}$ yielding essentially identical melting profiles, but with apparent $T_{\mathrm{m}}$ values shifted to higher temperatures (data not shown and Table 1). Thus, a dramatic difference in stability between the two conformers of nc $886\left(\Delta T_{\mathrm{m}}>30^{\circ} \mathrm{C}\right)$ was observed under both the lower and higher ionic strength conditions. A mixture of the conformers, purified using standard denaturing PAGE, was also analyzed under both solution conditions and had a melting profile consistent with a combination of the profiles for the two individual conformers (Fig. 2A).

Collectively, these observations demonstrate that the purified nc886 conformers retain their conformational identity under native conditions and adopt structures with very different stabilities. Interestingly, however, upon complete thermal denaturation (heating to $>90^{\circ} \mathrm{C}$ ) and refolding, a subsequent analysis reveals that Conformer 1 can either readopt its original structure or can refold as Conformer 2. In contrast, Conformer 2 exclusively re-adopts its original structure (Fig. 2B,C).

\section{nc886 RNA conformers have opposing activities against PKR}

To begin characterizing the activities of the nc 886 conformers, we first tested the ability of each isolated RNA to bind to 
A

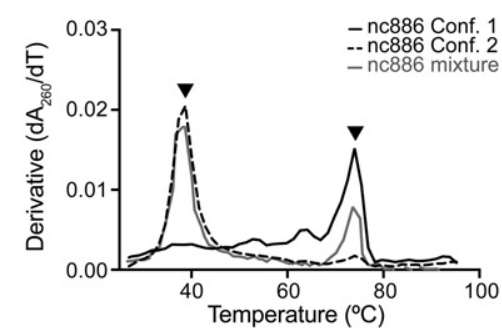

B

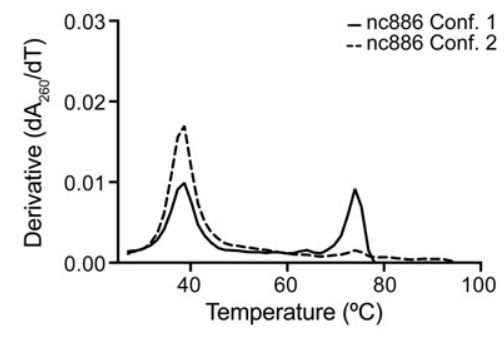

C

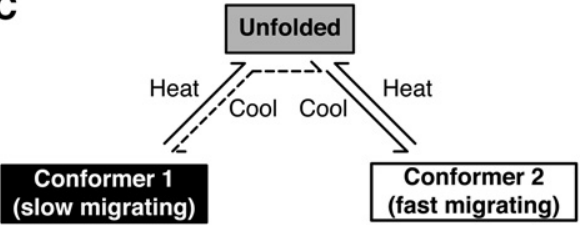

FIGURE 2. nc886 conformer stability and refolding. (A) UV melting profiles of each isolated native PAGE purified nc886 RNA conformer, and of a mixture of the two produced by denaturing PAGE purification. Melting temperatures $\left(T_{\mathrm{m}}\right)$ for the major apparent unfolding transitions (marked with arrowheads) are listed in Table 1. (B) UV melting profiles for each conformer of nc886 after complete thermal denaturation $\left(>90^{\circ} \mathrm{C}\right)$ and refolding. $(\mathrm{C})$ Schematic illustrating the observed interconversion properties of each nc886 conformer. Under native conditions both conformers are stable and do not interconvert (lower boxes). Following denaturation (Heat) and refolding (Cool), Conformer 1 can re-adopt its original structure or refold as Conformer 2 (dashed double arrow), while in contrast Conformer 2 only refolds into its original form.

PKR using an electrophoretic mobility shift assay (EMSA). Each individual purified conformer $(300 \mathrm{nM})$ was incubated with a range of PKR concentrations $(0-3 \mu \mathrm{M})$ and free and bound RNA resolved by native PAGE (Fig. 3A). Both conformers bind to PKR, but with starkly different affinities: while nc886 Conformer 1 readily forms a PKR-RNA complex, with shifted RNA observed at the lowest protein concentration, nc886 Conformer 2 does so only at the highest concentrations of PKR.

Each isolated nc886 conformer appears to result in a PKRnc886 complex with similar native gel mobility (Fig. 3A). We therefore considered the possibility that PKR binding might induce a structural conversion in one or both of the nc 886 conformers such that the final bound structure is the same regardless of the original nc886 conformation. Each nc886 conformer $(1 \mu \mathrm{M})$ was treated in an equivalent manner as for the EMSA analysis, either in the absence or presence of a twofold excess of PKR, except that the RNA was subsequently phenol: chloroform extracted and ethanol precipitated prior to analysis by native PAGE. Following this process, the original mobility of each nc886 RNA conformer was fully retained
(Fig. 3B), indicating that PKR binding does not induce a conformational interconversion in either nc886 conformer.

We next tested the effect that these differences in PKR binding had on the ability of each nc 886 conformer to inhibit PKR autophosphorylation in the presence of a dsRNA activator using an established slot-blot radiometric kinase assay (Sunita et al. 2015). PKR autophosphorylation was measured in the presence of a fixed concentration of poly(rI:rC) dsRNA and increasing amounts of each nc886 RNA conformer. Both conformers cause a dose-dependent decrease in PKR autophosphorylation, but to markedly different extents (Fig. 3C). nc886 Conformer 1 is a potent, dose-dependent inhibitor of PKR, capable of completely abolishing PKR autophosphorylation. In contrast, with Conformer 2 partial inhibition was only observed at the highest RNA concentration tested. Surprisingly, at the mid-range concentrations of nc886 RNA, where Conformer 1 fully inhibits PKR, Conformer 2 appears to modestly increase the extent of PKR autophosphorylation compared to poly(rI:rC) dsRNA alone. This prompted us to test whether nc886 Conformer 2 is capable of weakly activating PKR in the absence of dsRNA. Using an autophosphorylation assay in which PKR was incubated with increasing concentrations of nc886 Conformer 2 (0.001-10 $\mu \mathrm{M}$ ) alone, a dose-dependent increase in PKR autophosphorylation was indeed observed (Fig. 3D). Further, the extent of autophosphorylation was comparable to the amount of additional activation we observed in the inhibition assay (Fig. 3C, D). These results demonstrate that rather than simply being a poorer PKR inhibitor, nc886 RNA Conformer 2 behaves as a pseudoinhibitor. This contributes to overall PKR activation at lower concentrations, but competes for PKR binding with the more potent poly(rI:rC) dsRNA activator at the highest concentration in the inhibition assay.

\section{Differences in apical stem-loop structure distinguish the two nc886 RNA conformers}

To understand the basis for the stark differences in activity between nc886 conformers, we used selective 2'-hydroxyl

TABLE 1. Apparent melting temperatures $\left(T_{\mathrm{m}}\right)$ for wild-type and variant nc886 unfolding

\begin{tabular}{lcc}
\hline & \multicolumn{2}{c}{ Apparent $T_{\mathrm{m}}\left({ }^{\circ} \mathrm{C}\right)^{\mathrm{a}}$} \\
\cline { 2 - 3 } RNA & $0.5 \times \mathrm{TBE}$ & $\mathrm{HEPES} / \mathrm{NaCl}$ \\
\hline nc886 Conf. 1 & 73.8 & 92.2 \\
nc886 Conf. 2 & 38.2 & 58.4 \\
nc886 $\Delta$ TS Conf. 1 & 71.7 & 87.7 \\
nc886 $\Delta$ TS Conf. 2 & 31.8 & 52.8 \\
nc886 $\Delta$ AS & 44.4 & 61.6 \\
nc886 $\Delta$ L5 & 37.6 & 59.4 \\
\hline
\end{tabular}

${ }^{a}$ Value is for the major peak in the melting profile under each condition $(0.5 \times$ Tris-Borate-EDTA buffer or $20 \mathrm{mM}$ HEPES buffer $\mathrm{pH} 7.5$ containing $100 \mathrm{mM} \mathrm{NaCl}$ ). Estimated error for $T_{\mathrm{m}}$ values $\pm 0.5^{\circ} \mathrm{C}$. 
A

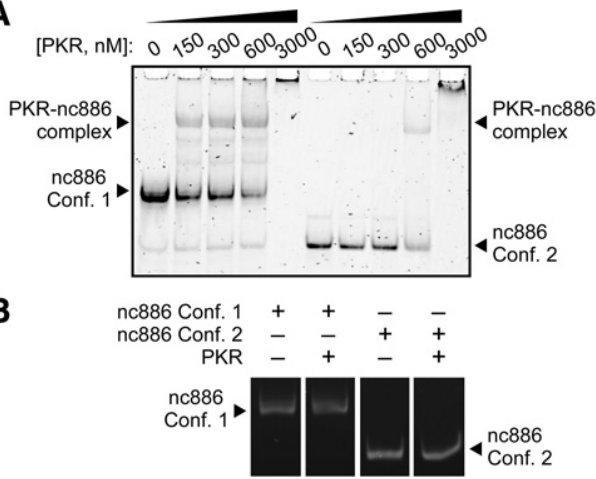

C

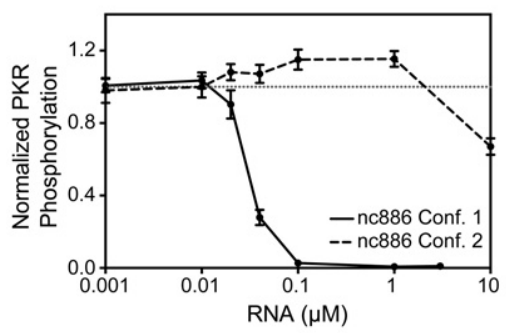

D

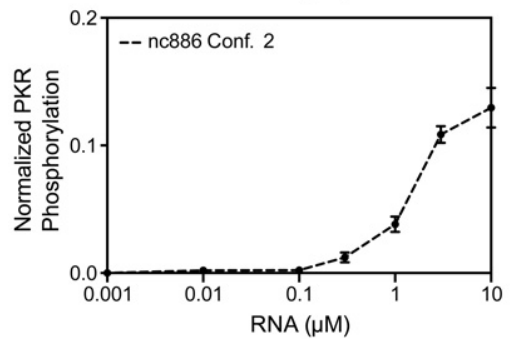

FIGURE 3. The individual nc886 conformers have distinct activities against PKR. (A) EMSA analysis of binding of each individual nc886 conformer to PKR. Native PAGE gels were stained with SYBR Green to identify free and PKR-bound RNA. (B) Native PAGE analysis of purified conformers extracted following incubation with or without PKR demonstrating that nc886 conformers do not undergo PKR binding-induced conformational interconversion. $(C)$ Quantification of slot-blot radiometric PKR autophosphorylation inhibition assays with each individual isolated nc886 conformer. The dotted line represents the extent of PKR phosphorylation, in the absence of any nc886 RNA, at the fixed concentration of poly(rI:rC) dsRNA activator used in all samples. (D) Radiometric PKR autophosphorylation activation assay demonstrating the capacity of nc886 Conformer 2 to weakly activate PKR.

acylation analyzed by primer extension (SHAPE) with $\mathrm{N}$ methylisotoic anhydride (NMIA) to experimentally assess the secondary structure of each isolated RNA. A $5^{\prime}$-end ${ }^{32} \mathrm{P}$-labeled DNA oligonucleotide complementary to the $3^{\prime}$ end of nc886 RNA was used for reverse transcription (RT) following incubation of each individual nc886 conformer with or without NMIA, and the products resolved on denaturing polyacrylamide sequencing gels (Fig. 4).

The SHAPE reactivity for Conformer 2 correlates well with the predicted secondary structure for nc886 RNA, with strong modification of most nucleotides within predicted loops and essentially none in predicted base-paired regions (Fig. 4A,C). The most significant deviation between SHAPE reactivity and the predicted structure occurs for the $5^{\prime}$-side of Loop 3 (nucleotides 25-28), which exhibits lower than expected reactivity, indicative of a more base-paired or rigid structure. Probing of nc886 Conformer 1 revealed an essentially identical pattern for the $3^{\prime}$-half of the RNA, with high reactivity within the $3^{\prime}$-side of Loop 3 and within Loop 4. However, in sharp contrast to Conformer 2, Loop 5 reactivity is absent in Conformer 1 except at the single, strongly reactive nucleotide A47 (Fig. 4C). Additionally, unique to Conformer 1, a series of remarkably strong, NMIA-independent stops was observed corresponding to the nucleotides immediately preceding Loop 5 in the structure (nucleotides 36-40; Fig. 4B,C), indicative of a stable structure resistant to unfolding during the RT reaction. The remainder of the $5^{\prime}$-half of Conformer 1 was examined using a second, internal primer (complementary to nucleotides 36-54) and found to have similar reactivity as Conformer 2 in this region, including the strong reactivity in the $5^{\prime}$-sides of Loop 1 and lower than expected reactivity for Loop 3.

These results demonstrate that each nc886 conformer adopts a common structure within its terminal and central regions, but that they differ dramatically in the apical stem-loop. The reduced reactivity of Loop 5 and the adjacent RT-read-through resistant sequence suggest that the apical stem-loop adopts a stable, higher order structure unique to nc886 Conformer 1 . As the only significant structural difference between the two nc886 conformers, these data strongly suggest that the absence (Conformer 2) or presence (Conformer 1) of this additional structural element is responsible for their different native gel motilities, thermal stabilities, and activities against PKR.

\section{The unique Conformer 1 apical stem-loop structure is critical for PKR repression}

We next sought to fully define which regions of nc886 are critical for binding and inhibition of PKR. Based on the SHAPE probing data, we created nc886 variants with a truncation of either the terminal stem (nc886 $\Delta$ TS) or the apical stem-loop (nc886 $\Delta$ AS) (Fig. 5A). The nc $886 \Delta$ TS variant exists as two conformers that possess similar relative stability to each full-length nc886 conformer as determined by UV thermal melting analysis (Table 1; Fig. 5B). These observations are consistent with the nc886 $\Delta$ TS conformers retaining a common central stem organization but distinct structures in the apical stem-loop as for the wild-type RNA. In contrast, nc886 $\Delta$ AS folds into a single conformer with an apparent $T_{\mathrm{m}}$ for unfolding most similar to Conformer 2 of full-length nc886 and nc886 $\Delta$ TS. Again, this result is consistent with the apical stem-loop containing the structure that distinguishes the two nc 886 RNA conformers. The nc $886 \Delta$ TS conformers and nc $886 \triangle \mathrm{AS}$ were native PAGE purified as previously described and the isolated RNAs tested for binding to PKR. The nc886 $\Delta$ TS conformers were found to have similar binding properties as their full-length nc886 equivalents (Fig. 5C), with nc886 $\Delta$ TS Conformer 1 binding PKR with 

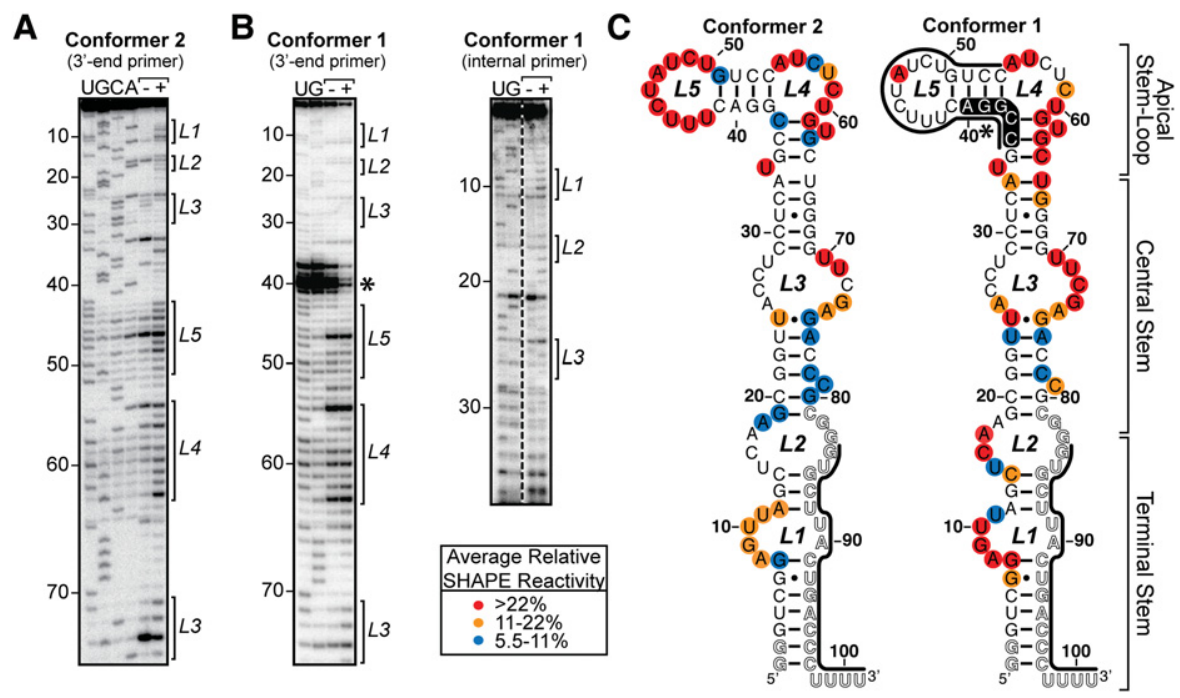

FIGURE 4. The apical stem-loop of nc886 distinguishes the two RNA conformers. (A) Example autoradiogram of sequencing gel analysis of nc886 Conformer 2 SHAPE probing. Lanes are:-, mock treated (no NMIA); +, NMIA treated; and, U/G/C/A, sequencing lanes containing the complementary dideoxy NTP. Brackets on the right of the gel image denote the loop regions (L1-L5) in the predicted nc886 RNA secondary structure. $(B)$ Example autoradiogram of sequencing gel analysis of nc886 Conformer 1 SHAPE probing using either the $3^{\prime}$-end (left) or internal (right) primer. Lane labels are the same as Panel $A$. (C) Categorized average nucleotide SHAPE reactivity for each conformer mapped onto the predicted nc886 secondary structure. Also noted are the sequences complementary to the $3^{\prime}$-end and internal primers (thick black lines), nucleotides for which reactivity could not be determined (outline font), and the strong structure-induced RT stops (nucleotides 36-40) observed only for nc886 Conformer 1 (black shading and region marked by an asterisk in panels $B$ and $C$ ).

higher affinity than Conformer 2. In contrast, nc886 $\triangle$ ASPKR binding was significantly reduced, comparable to the level of full-length nc886 and nc886 $\Delta$ TS Conformer 2.

We next tested the ability of each RNA variant to inhibit PKR autophosphorylation. Again, the activity of each nc $886 \Delta$ TS conformer closely resembled that of the equivalent full-length nc886 conformer, whereas nc886 $\triangle \mathrm{AS}$ was a poor PKR inhibitor, comparable to nc $886 \Delta$ TS Conformer 2 (Fig. 5D). Both nc $886 \Delta$ AS and nc $886 \Delta$ TS Conformer 2 were additionally tested in the kinase activation assay to determine whether these RNAs weakly activate PKR as for full-length nc886 Conformer 2. However, neither of these RNAs activated PKR at any of the concentrations tested (Fig. 5E). These results suggest that in the presence of poly(rI:rC) dsRNA, the similarly weak inhibition of PKR observed at the highest concentration of full-length nc886 Conformer 2, nc886 4 TS Conformer 2, and nc886 $\triangle$ AS RNAs arises due to their comparable weak PKR binding affinities. However, unlike fulllength nc886 Conformer 2 which weakly activates PKR in the absence of other dsRNA, nc886 $\Delta$ TS Conformer 2 has lost this ability. This result suggests that the nc886 terminal stem contributes significantly to this activity in the context of the structure of Conformer 2, which lacks the high affinity apical stem-loop PKR binding site.

The cellular impact of PKR activation is the downstream phosphorylation of its substrate eIF2 $\alpha$. We therefore tested the effect of full-length and each variant nc886 RNA on PKR's ability to phosphorylate eIF2 $\alpha$ using the same radiometric kinase assay, except that the phosphorylated protein products were resolved by SDS-PAGE prior to autoradiography. After a preincubation in the absence or presence of each nc886 RNA $(10 \mu \mathrm{M})$, the extent of PKR and eIF $2 \alpha$ phosphorylation was measured following incubation with poly(rI: rC) dsRNA activator (Fig. 6A). Full-length nc886 Conformer 1 and nc886 $\Delta$ TS Conformer 1 both potently inhibit PKR and downstream eIF2 $\alpha$ phosphorylation (Fig. 6B). In contrast, nc886 Conformer 2, nc886 $\Delta$ TS Conformer 2, and nc886 $\triangle$ AS only partially inhibit PKR autophosphorylation and, as a result, confer almost no inhibition of eIF2 $\alpha$ phosphorylation.

Collectively, the combined structural and functional analyses of wild-type and variant nc886 conformers point to a unique structure, present only in Conformer 1, being responsible for potent $\mathrm{PKR}$ inhibition. To this end, we created a final variant RNA (nc886 $\Delta$ L5) with a minimally altered Loop 5 sequence designed to specifically disrupt this apical stem-loop structure. SHAPE probing and native PAGE analysis demonstrates that this variant RNA exists as a single conformer that adopts a secondary structure similar to wild-type Conformer 2 (Fig. 7A,B). UV thermal melting analysis indicates the stability of the RNA to be similar to that of wild-type Conformer 2 (Table 1). We next tested for PKR binding using the same electrophoretic mobility shift assay and found that nc886 $\triangle$ L5 binds PKR weakly, similar to both wild-type nc886 Conformer 2 and the larger $\triangle$ AS truncation variant (Fig. 7C). Finally, we tested nc886 $\Delta$ L5 for its ability to inhibit PKR autophosphorylation in the presence of the synthetic dsRNA poly(rI:rC) using the radiometric kinase assay. 
A
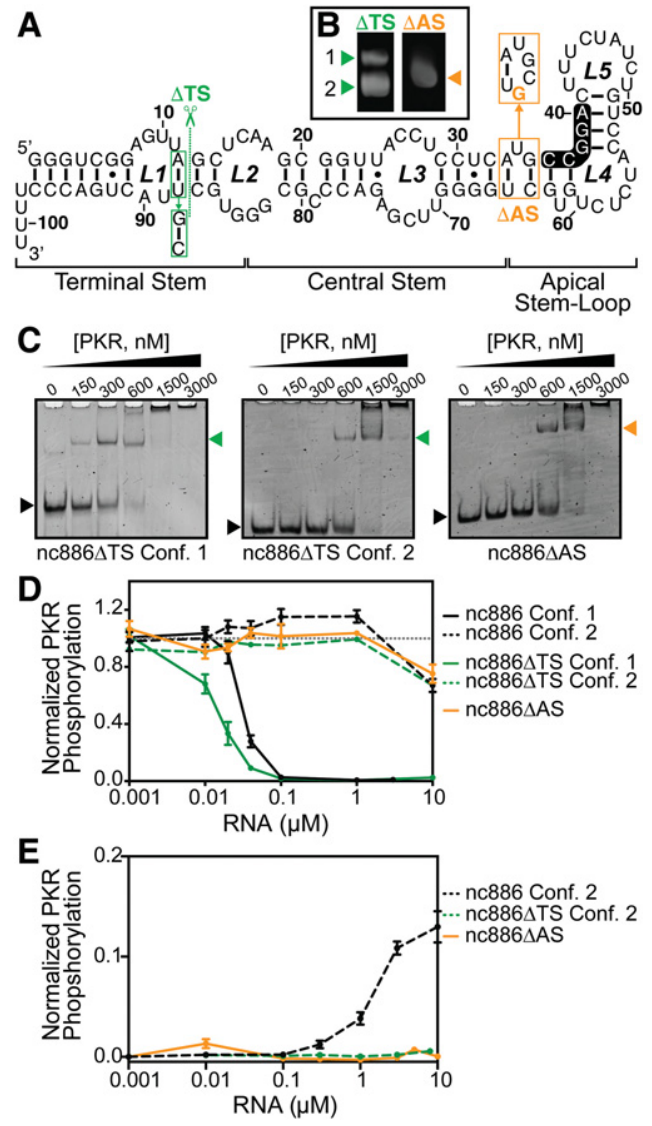

FIGURE 5. Binding and inhibition of PKR by terminal stem and apical stem-loop deletion variants of nc886 RNA. (A) Schematic showing the RNA regions and sites of truncation for the nc886 terminal stem and the apical stem-loop to create variants nc $886 \Delta \mathrm{TS}$ (deletion of nucleotides 1-12 and 88-101) and nc886 $\triangle$ AS (deletion of nucleotides 36-63), respectively. One additional sequence alteration ( $G$ insertion) in nc886 $\triangle \mathrm{AS}$ is highlighted within the orange dashed line boxes. $(B)$ Native PAGE analysis of in vitro transcribed variant nc886 RNAs. nc $886 \Delta \mathrm{TS}$, but not nc $886 \Delta$ AS, maintains formation of two conformers. (C) EMSA analysis of RNA variants binding to PKR for nc886 $\Delta$ TS Conformer 1, nc886 $\Delta$ TS Conformer 2, and nc886 $\Delta$ AS RNAs. Native PAGE gels were stained with SYBR Green to identify free (black arrows) and PKR-bound RNA (green or orange). (D) Quantification of slot-blot radiometric PKR autophosphorylation inhibition assays with nc $886 \Delta$ TS Conformer 1 (solid green line), nc886 $\Delta$ TS Conformer 2 (dashed green line), and nc $886 \Delta \mathrm{AS}$ (solid orange line). Full-length nc 886 data (black lines) shown for comparison are the same as those shown in Figure 3C. The dotted line represents the extent of PKR phosphorylation, in the absence of any nc886 RNA, at the fixed concentration of poly(rI: rC) dsRNA activator used in all samples. (E) Radiometric PKR autophosphorylation activation assay demonstrating the loss of PKR activation in nc886 $\triangle \mathrm{AS}$ (solid orange line) and nc886 $\Delta$ TS Conformer 2 (dashed green line), as compared to full-length nc886 Conformer 2 (dashed black line). nc886 Conformer 2 data are the same as those shown in Figure 3D.

nc886 $\triangle \mathrm{L} 5$ is a poor PKR inhibitor (Fig. 7D), consistent with Loop 5 being critical for forming the apical stem-loop structure essential for potent PKR inhibition. Contrary to our expectation, however, nc886 $\Delta \mathrm{L} 5$ did not retain the capacity of wild-type Conformer 2 to weakly activate PKR. This suggests the full apical stem-loop structure of the RNA is required in addition to the terminal stem for this activity.

\section{DISCUSSION}

PKR binds to and is activated by RNAs containing a variety of structural elements, including internal bulges, stem-loops, and single-stranded regions, or RNAs which may only be sufficiently double-stranded through tertiary interactions (Osman et al. 1999; Nussbaum 2002; Kim et al. 2006; Cohen-Chalamish et al. 2009; Heinicke et al. 2009; Nallagatla et al. 2011; Dzananovic et al. 2013; Mayo et al. 2016). With our growing appreciation that cellular RNAs adopt complex secondary and tertiary folds, the number of potential PKR activators in a cell is high. The identification that nc886 RNA functions as a negative regulator of PKR (Lee et al. 2011) thus provides a cellular mechanism to repress spurious inhibition of translation in the absence of viral infection.

In this study, we have demonstrated that nc886 RNA can adopt two nonequivalent structures that modulate PKR activity in distinct and opposing manners. Our SHAPE structure probing studies revealed that Conformer 2 adopts an apical stem-loop structure consistent with the predicted secondary structure for nc886, specifically, high reactivity of all Loop 5 nucleotides and low reactivity for the nucleotides of the adjacent four base pair helix. In contrast, the Loop 5 nucleotides of nc886 Conformer 1 had predominantly low reactivity, and the $5^{\prime}$-half of the adjacent helix was found to block reverse transcriptase, independent of the presence of SHAPE probing reagent. RNA dimerization is known to influence PKR-RNA interaction, resulting in increased PKR

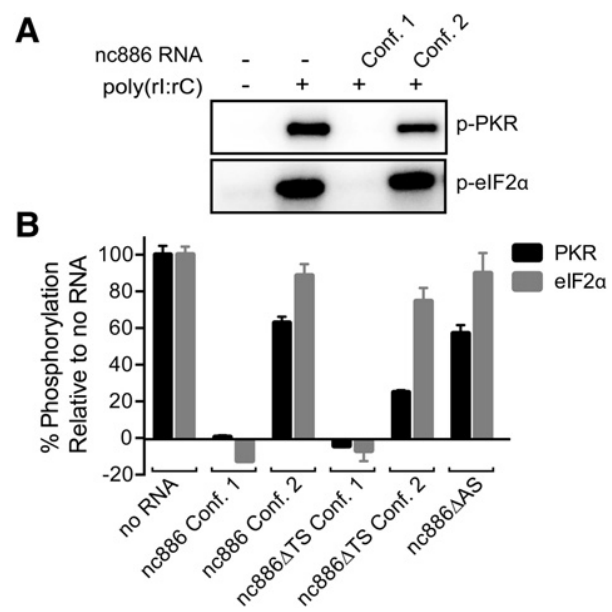

FIGURE 6. Inhibition of PKR phosphorylation of eIF2 $\alpha$ substrate by wild-type and nc886 RNA variants. (A) Example SDS-PAGE analysis of a radiometric kinase inhibition assay containing both PKR and eIF2 $\alpha$ substrate. (B) Quantification of PKR and eIF2 $\alpha$ phosphorylation in the presence of fixed concentrations of poly $(\mathrm{rI}: \mathrm{rC}) \mathrm{RNA}(0.05 \mu \mathrm{g} / \mathrm{mL})$ and the indicated nc886 RNA variant $(10 \mu \mathrm{M})$ normalized to a no RNA control after background subtraction. 


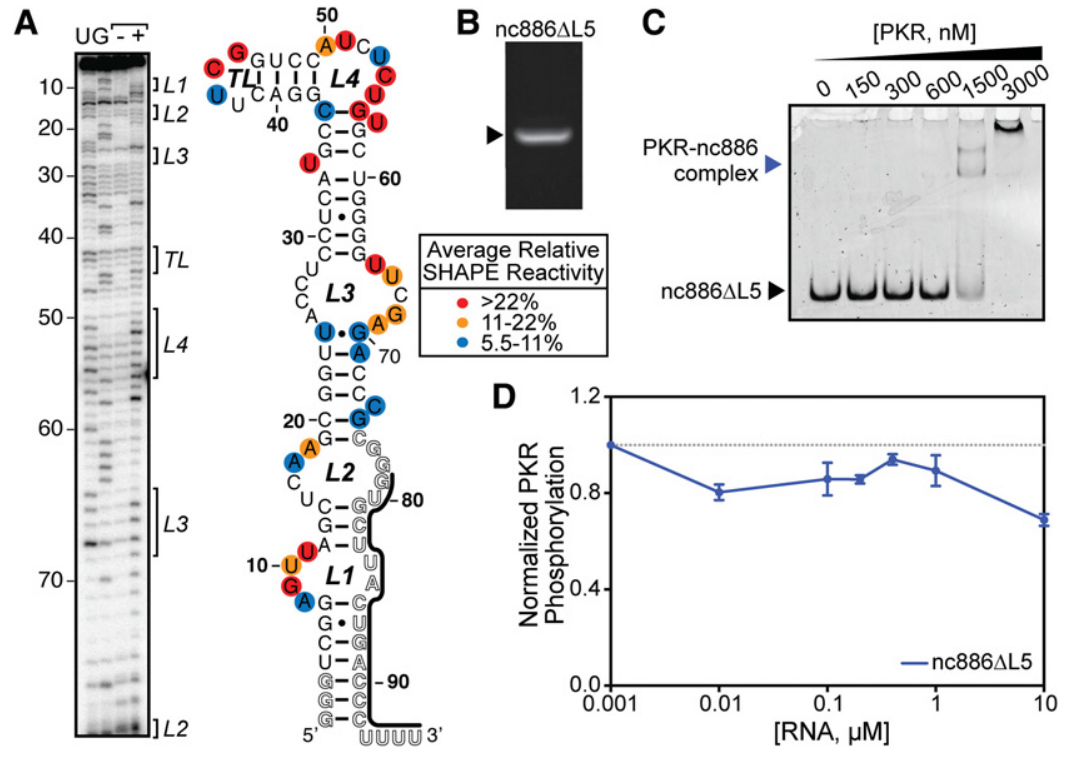

FIGURE 7. Alteration of loop 5 (nucleotides 44-48; nc886 $\Delta$ L5) disrupts the structure specific to nc886 Conformer 1 and abrogates PKR inhibition. (A) Left, example autoradiogram of sequencing gel analysis of nc886 $\triangle \mathrm{L} 5 \mathrm{SHAPE}$ probing. Lanes are: -, mock treated (no NMIA); +, NMIA treated; and, U/G, sequencing lanes containing the complementary dideoxy NTP. Brackets on the right of the gel image denote the loop regions (L1 to TL) in the predicted nc886 RNA secondary structure. Right, categorized average nucleotide SHAPE reactivity mapped onto the predicted secondary structure. (B) Native PAGE analysis of nc886 $\Delta$ L5 RNA. (C) EMSA analysis of nc886 $\Delta$ L5 RNA binding to PKR using SYBR Green staining to identify free (black arrow) and PKR-bound RNA (blue arrow). (D) Quantification of slot-blot radiometric PKR autophosphorylation inhibition assays with nc886 $\Delta \mathrm{L} 5$. The dotted line represents the extent of PKR phosphorylation, in the absence of any nc886 RNA, at the fixed concentration of poly(rI:rC) dsRNA activator used in all samples.

activation (Heinicke et al. 2009; Hull and Bevilacqua 2016). Dimerization could potentially give rise to the relative native gel mobilities of the two nc886 conformers and result in the changes in SHAPE reactivity we observed for Conformer 1. Dimerization-based generation of a PKR-inhibitory structure would be an unexpected mechanism of PKR regulation. However, the significantly greater stability of Conformer 1 and the equivalent native gel mobility of the PKR complexes with each conformer argue against nc886 dimerization as the difference between the two forms. Further, we have found that while neither nc886 conformer is formed in a concentration-dependent manner, both conformers form slower migrating bands on native gel at higher concentrations and both these dimeric/oligomeric forms can be disrupted by annealing at temperatures below the unfolding of Conformer 1 (data not shown). Thus, while dimerization cannot be definitively excluded, we propose based on our results that Conformer 1 adopts a higher order structure involving nucleotides of Loop 5 within its apical stem-loop and that this tertiary structure distinguishes the two monomeric forms of nc 886 .

nc886 Conformer 1 binds with higher affinity resulting in potent inhibition of PKR whereas Conformer 2 binds more weakly and, surprisingly, behaves as a pseudoinhibitor. As such, nc886 Conformer 2 can weakly activate the kinase but becomes inhibitory at high concentrations against a more potent activator, such as poly(rI: rC) dsRNA. These opposing functions also impact phosphorylation of PKR's substrate eIF2a: nc886 Conformer 1 effectively inhibits the downstream phosphorylation of eIF $2 \alpha$, while Conformer 2 results in no loss of eIF2 $\alpha$ phosphorylation. The two nc886 conformers adopt common terminal and central stem structures, and deletion of the terminal stem has no impact on the formation of distinct RNA conformers or the PKR binding and inhibitory potential of each compared to the wild-type RNA. In contrast, deletion of the entire apical stemloop or just Loop 5 results in a single conformer with the same functional properties as Conformer 2. These observations demonstrate the critical importance of the unique apical stem-loop structure of Conformer 1 for potent nc886-mediated inhibition of PKR. However, highresolution structural analysis will be required to fully define the nc886 Conformer 1 apical stem-loop structure and thus reveal the basis for its specific ability to potently inhibit PKR. Furthermore, the nature of the two nc886 conformers has direct functional consequences for PKR regulation, and it may not be appropriate to simply consider them as active and inert forms, as previously suggested (Jeon et al. 2012).

Aside from its central importance to innate immunity, PKR plays diverse roles in cell signaling pathways and during mitosis, cell differentiation, and transcriptional regulation (García et al. 2006, 2007; Kang and Tang 2012; Liu et al. 2013; Kim et al. 2014; Youssef et al. 2015). Thus, there is a clear need for tight regulation of PKR activity in the uninfected cell in a manner which still allows for appropriate activation by cellular RNAs and proteins. Identification of opposing PKR-regulatory activities for the two nc886 conformers suggests a potential mechanism that would allow for a gradient of regulation rather than all-or-nothing PKR inhibition. We speculate that by tuning the levels of each nc886 conformer, the cell would be able to prevent basal activation of PKR in the absence of infection while still allowing for specific and timed activation in response to diverse cellular needs. The nc886 conformer levels and/or ratio could be shifted either transcriptionally or through refolding of nc886, similar to the autoregulation of IFN- $\gamma$ mRNA translation via refolding of its $5^{\prime}$-untranslated region into a PKR-activating structure (Cohen-Chalamish et al. 2009). During viral infection, either conformer of nc886 RNA could be easily displaced by viral RNAs through greater binding affinity or through excess viral dsRNA, thus allowing PKR to mount 
an immune response. Indeed, in cells stably expressing nc886, poly(rI:rC) dsRNA transfection still elicits PKR activation (Jeon et al. 2012). The questions of if, how, and when the levels or ratios of the nc886 conformers may change during the life of the cell remain to be answered.

The importance of nc886 in the regulation of PKR activity is highlighted by both its implication in disease and by the perturbation of its function by a range of viruses (García et al. 2006; Langland et al. 2006; Roberts et al. 2009; Marchal et al. 2014). Addressing what other cellular binding partners nc886 RNA may have and whether these would be shared or distinct between the two conformers would reveal potential mechanisms by which nc886 dysregulation might contribute to disease. Moreover, viruses also inhibit PKR activity through the actions of nc886. For example, EpsteinBarr virus infection stimulated a three- to fourfold increase in the level of nc886 RNA (then named CBL3) (Nandy et al. 2009) and Influenza A virus infection increased levels of nc886 (then named vtRNA 2-1) by 25-fold ( $\mathrm{Li}$ et al. 2015). However, whether viruses may also influence the relative expression of the nc886 conformers to promote their replication by masking detection for survival is unknown.

In summary, the current study has revealed new insights into how nc886 RNA adopts multiple conformers that are critical for its function as a regulator of PKR activity, as well as elucidating the differences both in their structure and function. Future studies building on this work would contribute to our understanding of this critical regulator of innate immunity and could potentially inform therapeutic approaches to inhibit or to stimulate the immune response through the actions of this noncoding RNA.

\section{MATERIALS AND METHODS}

\section{RNA in vitro transcription and purification}

Full-length and variant nc886 RNAs were in vitro transcribed from linearized plasmid DNA templates using T7 RNA polymerase as previously described (Linpinsel and Conn 2012). nc886 variants were produced in the same way from plasmids encoding sequences with the following changes: deletion of nucleotides 1-12 and 88-101 (nc886 $\Delta$ TS), deletion of nucleotides 36-63 (nc886 $\triangle A S$ ), and deletion of nucleotides $44-48$ with additional U50 to $\mathrm{G}$ mutation (nc886 $\Delta$ L5). Following dialysis against $1 \times$ Tris-EDTA (TE) buffer, RNAs were purified by polyacrylamide gel electrophoresis (PAGE) on denaturing ( $50 \%$ urea, $1 \times$ Tris-Borate-EDTA [TBE]) or native $(0.5 \times \mathrm{TBE})$ gels. RNA bands were identified by UV shadowing, excised, eluted from the gel by crushing and soaking in $0.3 \mathrm{M}$ sodium acetate, and ethanol precipitated as previously described (Linpinsel and Conn 2012).

\section{PKR and elF2 $\alpha$ protein expression and purification}

For PKR expression, E. coli Rosetta 2 (DE3) cells were transformed with the pET-PKR/PPase plasmid encoding full-length human PKR (Lemaire et al. 2008). Single colonies were used to inoculate large-scale cultures in Terrific Broth. PKR expression was induced with $0.1 \mathrm{mM}$ isopropyl $\beta$-D-1-thiogalactopyranoside (IPTG) at mid-log phase growth $\left(\mathrm{OD}_{600} \sim 0.5\right)$ and growth continued overnight at $20^{\circ} \mathrm{C}$. PKR was purified by sequential heparin-affinity (HiPrep Heparin 16/10), poly(rI:rC) dsRNA-affinity, and gel filtration (Superdex 200 10/300) chromatographies on an ÄKTApurifier10 system (GE Healthcare). PKR was eluted from the gel filtration column in $20 \mathrm{mM}$ HEPES buffer ( $\mathrm{pH}$ 7.5) containing $150 \mathrm{mM} \mathrm{NaCl}$, $0.1 \mathrm{mM}$ EDTA, $10 \%$ glycerol, and $10 \mathrm{mM} \beta$-mercaptoethanol (BME).

For eIF2a expression, E. coli BL21 (DE3) cells were transformed with a plasmid encoding an amino-terminal hexa-histidine-tagged human eIF2 $\alpha$. Single colonies were used to inoculate large-scale cultures in Lysogeny Broth. eIF2a expression was induced with $0.1 \mathrm{mM}$ IPTG at mid-log phase growth $\left(\mathrm{OD}_{600} \sim 0.5\right)$ and growth continued overnight at $18^{\circ} \mathrm{C}$. eIF2 $\alpha$ was purified using a bench-top HisSpinTrap column (GE Healthcare). Fractions containing the protein were pooled and further purified by gel filtration chromatography (Superdex 200 10/300) on an ÄKTApurifier10 system. eIF2 $\alpha$ was eluted from the gel filtration column in $20 \mathrm{mM}$ HEPES buffer ( $\mathrm{pH} 7.5$ ) containing $150 \mathrm{mM} \mathrm{NaCl}, 0.1 \mathrm{mM}$ EDTA, $10 \%$ glycerol, and $10 \mathrm{mM} \mathrm{BME}$.

\section{RNA UV thermal melting analysis}

RNA UV melting curves were collected at 260 and $280 \mathrm{~nm}$ on a Cary400 UV-visible spectrophotometer (Varian). Samples contained $20-25 \mu \mathrm{g}$ of RNA in a solution of either $0.5 \times$ TBE or 20 mM HEPES buffer ( $\mathrm{pH}$ 7.5) containing $100 \mathrm{mM} \mathrm{NaCl}$. To simplify comparisons between RNA constructs, the first derivative of each UV absorbance curve (the "melting profile") was calculated for each RNA in GraphPad Prism6 software after normalization using the following equation: $\left(\mathrm{Abs}_{\mathrm{T}}-\mathrm{Abs}_{\mathrm{Tmin}}\right) / \mathrm{Abs}_{\mathrm{Tmin}}$.

\section{Electrophoretic mobility shift assays (EMSA)}

A range of PKR concentrations, from $0-3 \mu \mathrm{M}$, was incubated with $300 \mathrm{nM}$ full-length or variant nc886 RNA for $30 \mathrm{~min}$ on ice in 20 mM HEPES ( $\mathrm{pH} 7.5$ ) $150 \mathrm{mM} \mathrm{NaCl}, 10 \%$ glycerol, and $2 \mathrm{mM}$ EDTA. After incubation, free and bound RNAs were resolved by native PAGE (10\% acrylamide, $0.5 \times \mathrm{TBE})$. Gels were stained with SYBR Green gel stain for $20 \mathrm{~min}$ and visualized on a Typhoon FLA 7000 (GE Healthcare) using the fluorescence setting and a $520 \mathrm{~nm}$ emission filter. EMSAs were repeated at least two times producing essentially identical results.

\section{PKR inhibition assays}

PKR $(0.1 \mu \mathrm{g})$ was preincubated with $0-10 \mu \mathrm{M}$ of full-length or variant nc886 RNA for $10 \mathrm{~min}$ at $25^{\circ} \mathrm{C}$ in $50 \mathrm{mM}$ Tris buffer ( $\mathrm{pH} 7.8$ ) containing $50 \mathrm{mM} \mathrm{KCl}, 2.5 \mathrm{mM}$ DTT, and 10\% glycerol. Reactions were initiated by the addition of $0.05 \mu \mathrm{g} / \mathrm{mL}$ poly(rI:rC) dsRNA, 20 $\mu \mathrm{M}$ ATP, $1 \mu \mathrm{Ci}\left[\gamma^{32} \mathrm{P}\right]$-ATP, and $2 \mathrm{mM} \mathrm{MgCl}_{2}$. After incubation at $25^{\circ} \mathrm{C}$ for $10 \mathrm{~min}$, reactions were quenched with excess ice-cold phosphate buffered saline containing $200 \mu \mathrm{M}$ ATP and applied to a BioDot SF (Bio-Rad) microfiltration system as described previously (Sunita et al. 2015). Membranes were exposed to a phosphor storage screen and the extent of phosphorylation was determined by analysis 
on a Typhoon FLA 7000 PhosphorImager and ImageQuant software (GE Healthcare). Assays were repeated at least two times for each RNA and normalized to a control lacking nc886 RNA after background subtraction.

Assays of inhibition of eIF $2 \alpha$ phosphorylation were carried out as described above but at a single nc886 RNA concentration $(10 \mu \mathrm{M})$ and with the additional inclusion of $0.25 \mu \mathrm{g}$ eIF $2 \alpha$ in the reaction initiation mixture. Reactions were quenched by the addition of gel loading dye for SDS-PAGE analysis. Gels were dried and exposed to a phosphor storage screen and analyzed as above. Assays of PKR and eIF2 $\alpha$ inhibition were repeated at least three times for each RNA. Phosphorylation intensity for each protein was normalized to a reaction lacking nc886 after background subtraction from a control reaction lacking both poly(rI:rC) dsRNA and nc886.

\section{PKR activation assays}

PKR $(0.1 \mu \mathrm{g})$ was incubated with $0-10 \mu \mathrm{M}$ of full-length or variant nc886 RNA for $10 \mathrm{~min}$ at $25^{\circ} \mathrm{C}$ in $50 \mathrm{mM}$ Tris buffer ( $\mathrm{pH} 7.8$ ) containing $50 \mathrm{mM} \mathrm{KCl}, 2.5 \mathrm{mM}$ DTT, 10\% glycerol, $20 \mu \mathrm{M}$ ATP, $1 \mu \mathrm{Ci}$ $\left[\gamma^{32} \mathrm{P}\right]$-ATP, and $2 \mathrm{mM} \mathrm{MgCl}_{2}$. After incubation, reactions were quenched with excess ice-cold phosphate buffered saline containing $200 \mu \mathrm{M}$ ATP and applied to a Bio-Dot SF (Bio-Rad) microfiltration system as described previously (Sunita et al. 2015). Membranes were exposed to a phosphor storage screen and the extent of phosphorylation was determined using analysis by a Typhoon FLA 7000 PhosphorImager and ImageQuant software (GE Healthcare). Values were normalized to a poly $(\mathrm{rI}: \mathrm{rC})(0.1 \mu \mathrm{g} / \mathrm{mL})$ reaction performed in parallel, following background subtraction from a control reaction without nc886 RNA. Assays were repeated at least three times for each RNA.

\section{Selective $2^{\prime}$-hydroxyl acylation analyzed by primer extension (SHAPE)}

SHAPE RNA probing with NMIA was carried out as described previously (Wilkinson et al. 2006) with the following modifications: Reactions were initiated using $1 \mu \mathrm{L}$ of $130 \mathrm{mM}$ NMIA for $45 \mathrm{~min}$ at $37^{\circ} \mathrm{C}$. Reverse transcription was carried out with a $5^{\prime}$-end labeled $\left[\gamma^{32} \mathrm{P}\right]$-ATP DNA primer corresponding to the sequence of the $3^{\prime}-$ end of the RNA (nucleotides 84-101 for both conformers) or to an internal sequence (nucleotides 36-54 for Conformer 1 only). To determine the position of each SHAPE reactive nucleotide, dideoxy nucleotide (ddNTP) sequencing was carried out using the radiolabeled primer and untreated RNA. All reactions were run on sequencing gels, dried, and exposed to a phosphor storage screen. The intensity of bands was analyzed on a Typhoon Trio Imager and quantified using ImageQuant software (GE Healthcare). Following subtraction of background reactivity in the no NMIA lanes, reactivity at each nucleotide was normalized and the values from at least two replicates were averaged and classified as $5.5 \%-11 \%, 11 \%-$ $22 \%$, and $>22 \%$ (Wilkinson et al. 2006). SHAPE reactivity for nc886 Conformer 1 was separately normalized to highest reactivity in each data set for the external and internal primers.

\section{ACKNOWLEDGMENTS}

We thank Dr. Christine M. Dunham and Samantha L. Schwartz for their comments on the manuscript and the members of the Conn and Dunham groups for useful discussions during the course of this work. This work was supported by a Bridge Funding Award from the Emory University School of Medicine and the Department of Biochemistry, and by the Biochemistry, Cell and Molecular Biology (BCMB) National Institutes of Health/National Institute of General Medical Sciences training grant T32-GM008367.

Received December 8, 2016; accepted January 3, 2017.

\section{REFERENCES}

Balachandran S, Barber GN. 2007. PKR in innate immunity, cancer, and viral oncolysis. Methods Mol Biol 383: 277-301.

Cao J, Song Y, Bi N, Shen J, Liu W, Fan J, Sun G, Tong T, He J, Shi Y, et al. 2013. DNA methylation-mediated repression of miR-886-3p predicts poor outcome of human small cell lung cancer. Cancer Res 73: 3326-3335.

Cohen-Chalamish S, Hasson A, Weinberg D, Namer LS, Banai Y, Osman F, Kaempfer R. 2009. Dynamic refolding of IFN- $\gamma$ mRNA enables it to function as PKR activator and translation template. Nat Chem Biol 5: 896-903.

Dalet A, Gatti E, Pierre P. 2015. Integration of PKR-dependent translation inhibition with innate immunity is required for a coordinated anti-viral response. FEBS Lett 589: 1539-1545.

Domingo-Gil E, Toribio R, Nájera JL, Esteban M, Ventoso I. 2011. Diversity in viral anti-PKR mechanisms: a remarkable case of evolutionary convergence. PLoS One 6: e16711.

Dzananovic E, Patel TR, Deo S, McEleney K, Stetefeld J, McKenna SA. 2013. Recognition of viral RNA stem-loops by the tandem doublestranded RNA binding domains of PKR. RNA 19: 333-344.

García MA, Gil J, Ventoso I, Guerra S, Domingo E, Rivas C, Esteban M. 2006. Impact of protein kinase PKR in cell biology: from antiviral to antiproliferative action. Microbiol Mol Biol Rev 70: 1032-1060.

García MA, Meurs EF, Esteban M. 2007. The dsRNA protein kinase PKR: virus and cell control. Biochimie 89: 799-811.

Heinicke LA, Wong CJ, Lary J, Nallagatla SR, Diegelman-Parente A, Zheng X, Cole JL, Bevilacqua PC. 2009. RNA dimerization promotes PKR dimerization and activation. J Mol Biol 390: 319-338.

Hull CM, Bevilacqua PC. 2016. Discriminating self and non-self by RNA: roles for RNA structure, misfolding, and modification in regulating the innate immune sensor PKR. Acc Chem Res 49: $1242-1249$.

Iwasaki A. 2012. A virological view of innate immune recognition. Annu Rev Microbiol 66: 177-196.

Jeon SH, Lee K, Lee KS, Kunkeaw N, Johnson BH, Holthauzen LMF, Gong B, Leelayuwat C, Lee YS. 2012. Characterization of the direct physical interaction of nc886, a cellular non-coding RNA, and PKR. FEBS Lett 586: 3477-3484.

Kang R, Tang D. 2012. PKR-dependent inflammatory signals. Sci Signal 5: pe47.

Kim I, Liu CW, Puglisi JD. 2006. Specific recognition of HIV TAR RNA by the dsRNA binding domains (dsRBD1-dsRBD2) of PKR. J Mol Biol 358: 430-442.

Kim Y, Lee JH, Park JE, Cho J, Yi H, Kim VN. 2014. PKR is activated by cellular dsRNAs during mitosis and acts as a mitotic regulator. Genes Dev 28: 1310-1322.

Kunkeaw N, Jeon SH, Lee K, Johnson BH, Tanasanvimon S, Javle M, Pairojkul C, Chamgramol Y, Wongfieng W, Gong B, et al. 2012. Cell death/proliferation roles for nc886, a non-coding RNA, in the protein kinase $\mathrm{R}$ pathway in cholangiocarcinoma. Oncogene 32: 3722-3731.

Langland JO, Cameron JM, Heck MC, Jancovich JK, Jacobs BL. 2006. Inhibition of PKR by RNA and DNA viruses. Virus Res 119: 100-110.

Lee YS. 2015. A novel type of non-coding RNA, nc886, implicated in tumor sensing and suppression. Genomics Inform 13: 26-30.

Lee K, Kunkeaw N, Jeon SH, Lee I, Johnson BH, Kang GY, Bang JY, Park HS, Leelayuwat C, Lee YS. 2011. Precursor miR-886, a novel 
noncoding RNA repressed in cancer, associates with PKR and modulates its activity. RNA 17: 1076-1089.

Lee HS, Lee K, Jang HJ, Lee GK, Park JL, Kim SY, Kim SB, Johnson BH, Zo JI, Lee JS, et al. 2014a. Epigenetic silencing of the non-coding RNA nc886 provokes oncogenes during human esophageal tumorigenesis. Oncotarget 5: 3472-3481.

Lee KS, Park JL, Lee K, Richardson LE, Johnson BH, Lee HS, Lee JS, Kim SB, Kwon OH, Song KS, et al. 2014b. nc886, a non-coding RNA of anti-proliferative role, is suppressed by CpG DNA methylation in human gastric cancer. Oncotarget 5: 3944-3955.

Lemaire PA, Anderson E, Lary J, Cole JL. 2008. Mechanism of PKR activation by dsRNA. J Mol Biol 381: 351-360.

Li F, Chen Y, Zhang Z, Ouyang J, Wang Y, Yan R, Huang S, Gao GF, Guo G, Chen JL. 2015. Robust expression of vault RNAs induced by influenza A virus plays a critical role in suppression of PKR-mediated innate immunity. Nucleic Acids Res 43: 10321-10337.

Linpinsel JL, Conn GL. 2012. General protocols for preparation of plasmid DNA template, RNA in vitro transcription, and RNA purification by denaturing PAGE. In Recombinant and in vitro RNA synthesis, pp. 43-58. Springer Science+Business Media, NY.

Liu X, Bennett RL, Cheng X, Byrne M, Reinhard MK, May WS. 2013. PKR regulates proliferation, differentiation, and survival of murine hematopoietic stem/progenitor cells. Blood 121: 3364-3374.

Marchal JA, Lopez GJ, Peran M, Comino A, Delgado JR, GarciaGarcia JA, Conde V, Aranda FM, Rivas C, Esteban M, et al. 2014. The impact of PKR activation: from neurodegeneration to cancer. FASEB J 28: 1965-1974.

Mayo CB, Wong CJ, Lopez PE, Lary JW, Cole JL. 2016. Activation of PKR by short stem-loop RNAs containing single-stranded arms. RNA 22: 1065-1075.

McKenna SA, Lindhout DA, Shimoike T, Aitken CE, Puglisi JD. 2007. Viral dsRNA inhibitors prevent self-association and autophosphorylation of PKR. J Mol Biol 372: 103-113.

Nallagatla SR, Toroney R, Bevilacqua PC. 2011. Regulation of innate immunity through RNA structure and the protein kinase PKR. Curr Opin Struct Biol 21: 119-127.

Nandy C, Mrazek J, Stoiber H, Grasser FA, Huttenhofer A, Polacek N. 2009. Epstein-Barr virus-induced expression of a novel human vault RNA. J Mol Biol 388: 776-784.

Nussbaum JM. 2002. The 3'-untranslated regions of cytoskeletal muscle mRNAs inhibit translation by activating the double-stranded RNAdependent protein kinase PKR. Nucleic Acids Res 30: 1205-1212.

Osman F, Jarrous N, Ben-Asouli Y, Kaempfer R. 1999. A cis-acting element in the $3^{\prime}$-untranslated region of human TNF- $\alpha$ mRNA renders splicing dependent on the activation of protein kinase PKR. Genes Dev 13: 3280-3293.

Roberts LO, Jopling CL, Jackson RJ, Willis AE. 2009. Viral strategies to subvert the mammalian translation machinery. Prog Mol Biol Transl Sci 90: 313-367.

Romanelli V, Nakabayashi K, Vizoso M, Moran S, Iglesias-Platas I, Sugahara N, Simon C, Hata K, Esteller M, Court F, et al. 2014. Variable maternal methylation overlapping the nc886/vtRNA2-1 locus is locked between hypermethylated repeats and is frequently altered in cancer. Epigenetics 9: 783-790.

Schlee M, Hartmann G. 2016. Discriminating self from non-self in nucleic acid sensing. Nat Rev Immunol 16: 566-580.

Schoggins JW, Rice CM. 2011. Interferon-stimulated genes and their antiviral effector functions. Curr Opin Virol 1: 519-525.

Sudhakar A, Ramachandran A, Ghosh S, Hasnain SE, Kaufman RJ, Ramaiah KV. 2000. Phosphorylation of serine 51 in initiation factor $2 \alpha($ IF $2 \alpha)$ promotes complex formation between eIF2 $\alpha(P)$ and eIF2B and causes inhibition in the guanine nucleotide exchange activity of eIF2B. Biochemistry 39: 12929-12938.

Sunita S, Schwartz SL, Conn GL. 2015. The regulatory and kinase domains but not the interdomain linker determine human doublestranded RNA-activated kinase (PKR) sensitivity to inhibition by viral non-coding RNAs. J Biol Chem 290: 28156-28165.

Treppendahl MB, Qiu X, Sogaard A, Yang X, Nandrup-Bus C, Hother C, Andersen MK, Kjeldsen L, Mollgaard L, Hellstrom-Lindberg E, et al. 2011. Allelic methylation levels of the noncoding VTRNA2-1 located on chromosome 5q31.1 predict outcome in AML. Blood 119: 206-216.

Wilkinson KA, Merino EJ, Weeks KM. 2006. Selective 2'-hydroxyl acylation analyzed by primer extension (SHAPE): quantitative RNA structure analysis at single nucleotide resolution. Nat Protoc 1: 1610-1616.

Xiong Y, Zhang L, Holloway AK, Wu X, Su L, Kebebew E. 2011. MiR886-3p regulates cell proliferation and migration, and is dysregulated in familial non-medullary thyroid cancer. PLoS One 6: e24717.

Yamasaki S, Anderson P. 2008. Reprogramming mRNA translation during stress. Curr Opin Cell Biol 20: 222-226.

Yoneyama M, Fujita T. 2010. Recognition of viral nucleic acids in innate immunity. Rev Med Virol 20: 4-22.

Youssef OA, Safran SA, Nakamura T, Nix DA, Hotamisligil GS, Bass BL. 2015. Potential role for snoRNAs in PKR activation during metabolic stress. Proc Natl Acad Sci 112: 5023-5028.

Zuker M. 2003. Mfold web server for nucleic acid folding and hybridization prediction. Nucleic Acids Res 31: 3406-3415. 

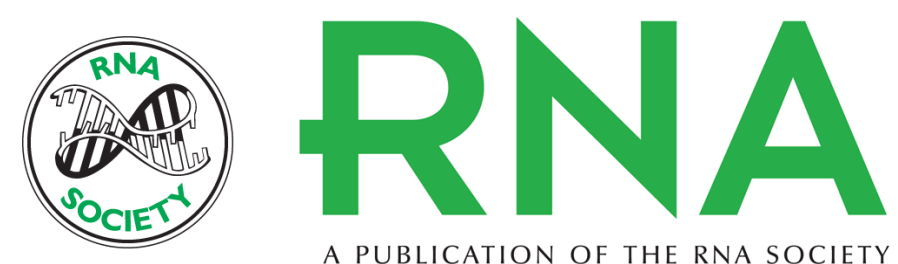

A PUBLICATION OF THE RNA SOCIETY

\section{Human noncoding RNA 886 (nc886) adopts two structurally distinct conformers that are functionally opposing regulators of PKR}

Brenda M. Calderon and Graeme L. Conn

RNA 2017 23: 557-566 originally published online January 9, 2017

Access the most recent version at doi:10.1261/rna.060269.116

References This article cites 44 articles, 10 of which can be accessed free at: http://rnajournal.cshlp.org/content/23/4/557.full.html\#ref-list-1

Creative This article is distributed exclusively by the RNA Society for the first 12 months after the Commons full-issue publication date (see http://rnajournal.cshlp.org/site/misc/terms.xhtml). After 12 License months, it is available under a Creative Commons License (Attribution-NonCommercial 4.0 International), as described at http://creativecommons.org/licenses/by-nc/4.0/.

Email Alerting Receive free email alerts when new articles cite this article - sign up in the box at the Service top right corner of the article or click here.

To subscribe to $R N A$ go to:

http://rnajournal.cshlp.org/subscriptions 